\title{
Development of a porcine skin injury model and characterization of the dose-dependent response to high-dose radiation
}

\author{
Jun Won $\mathrm{KIM}^{1}, \dagger$, Dong Won $\mathrm{LEE}^{2}, \dagger$, Won Hoon $\mathrm{CHOI}^{1}$, Yeo Reum JEON ${ }^{2}$, Se Hoon $\mathrm{KIM}^{3}$, \\ Haemi CHO ${ }^{1}$, Eun-Jung LEE ${ }^{1}$, Zhen-Yu HONG ${ }^{1}$, Won Jai LEE ${ }^{2}$ and Jaeho $\mathrm{CHO}^{1, *}$
}

${ }^{1}$ Department of Radiation Oncology, Yonsei University College of Medicine, 50 Yonsei-ro, Seodaemun-gu, Seoul, 120-752, South Korea

${ }^{2}$ Department of Plastic and Reconstructive Surgery, Yonsei University College of Medicine, 50 Yonsei-ro, Seodaemun-gu, Seoul, 120-752, South Korea

${ }^{3}$ Department of Pathology, Yonsei University College of Medicine, 50 Yonsei-ro, Seodaemun-gu, Seoul, 120-752,

South Korea

*Corresponding author. Department of Radiation Oncology, Severance Hospital, Yonsei University College of Medicine,

50 Yonsei-ro, Seodaemun-gu, Seoul, 120-752, South Korea. Tel: +82-2-2228-8095; Fax: +82-2-312-9033;

Email: jjhmd@yuhs.ac

${ }^{\dagger}$ These authors contributed equally to this work.

(Received 24 October 2012; revised 4 February 2013; accepted 5 February 2013)

\begin{abstract}
A porcine skin model was developed to characterize the dose-dependent response to high-dose radiation. The dorsal skin of a mini pig was divided into four paraspinal sections, with 11 small irradiation fields $(2 \mathrm{~cm} \times 2 \mathrm{~cm})$ in each section, and a single fraction of $15,30,50$ or $75 \mathrm{~Gy}$ was delivered to each section using a $6 \mathrm{MeV}$ electron beam. A spectrophotometer measured gross skin changes, and a biopsy for each radiation dose was performed in the 1st, 2nd, 4th, 6th and 9th weeks for histology, immunostaining with anti-CD31, and western blotting with IL- 6 and TGF- $\beta 1$ to determine the degree of skin damage. After a 4-week latency period, erythema and dry desquamation, moist desquamation, and ulceration appeared at 4, 6 and 9 weeks, respectively. Gross skin toxicity was more pronounced, occurred early and continued to progress with irradiation $>50 \mathrm{~Gy}$, whereas complete healing was observed 12 weeks after $15 \mathrm{~Gy}$. Spectrophotometry showed erythema indices rapidly increased during the first 4 weeks after irradiation. The number of eosinophils began rising sharply at 4 weeks and normalized after reaching peaks at 7-8 weeks. Microvessel density showed a biphasic pattern with a transient peak at 1 week, a nadir at 4-6 weeks, and maximum recovery at 9 weeks. Increase in the levels of IL- 6 and TGF- $\beta 1$ was detected soon after irradiation. Most of these parameters indicated complete healing of the skin 12 weeks after $15 \mathrm{~Gy}$. Our porcine skin model provides an effective platform for studying high-dose radiation-induced skin injury, in particular histologic and molecular changes, during the early latency period.
\end{abstract}

Keywords: radiation skin injury; porcine skin model; high-dose radiation

\section{INTRODUCTION}

Ionizing radiation causes tumor cell death as well as acute and late toxicities to the surrounding normal tissues. Because skin is usually the first site of entry in radiation treatment, variable degrees of skin reactions can occur. Patients who receive posterior neck electron boost for head and neck cancer, tumor bed boost for breast cancer, and postoperative radiotherapy (RT) for soft tissue sarcoma are often subjected to radiation higher than 60-70 Gy to the skin and experience moderate to severe skin reactions. Hypofractionated RT is regaining interest and an increasing number of patients with early cancer are undergoing stereotactic body radiotherapy (SBRT) with a hypofractionated schedule. However, there are concerns for increased acute and late toxicities that were not apparent with conventional schedules. Early reports from SBRT for early lung cancer and partial breast irradiation for early breast cancer have indicated increased skin toxicity [1,2]. 
Degrees and characteristics of radiation-induced skin injury depend on a variety of factors including total radiation dose, fractional dose, type and quality of radiation, and irradiated skin volume [3]. Serious radiation-induced skin injuries can cause severe pain, deformation, secondary infection, ulceration, and even necrosis when intolerable doses are administered [4], and quality of life for these patients is diminished considerably. Unfortunately, no preventive measure or effective treatment for radiation-induced skin injury is currently available except for conservative management. Thus, an effective strategy to prevent and treat radiation-induced skin injury is urgently needed in the modern RT era.

Unlike radiation treatment for human patients, who are often treated with multiple daily fractions over 6-7 weeks, irradiating a pig with multiple fractions of radiation is difficult, because daily generalized anesthesia may affect the pig's condition as well as the results of the study involving the pig. The most frequently used radiation schedule involving pig models is a single fraction of $\leq 20 \mathrm{~Gy}[2,5]$. In the current study, single fractional irradiation of $15 \mathrm{~Gy}, 30$ Gy, 50 Gy and 75 Gy was used in order to determine the dose-dependent response that could mimic the reactions of human skin that underwent multiple-fractioned radiation treatment of 60-70 Gy, as well as the maximum radiation dose that our pig skin model could tolerate without irreversible skin damage. The maximum tolerated dose will be used in further molecular studies and in developing modulating factors for radiation-induced skin injuries.

\section{MATERIALS AND METHODS}

\section{Porcine skin irradiation}

The study design was approved by the Institutional Animal Care and Use Committees. A female Micro-pig ${ }^{\circledR}$ (Medi Kinetics Co, Ltd., Pyeongtaek, Korea) weighing $25 \mathrm{~kg}$ was anesthetized with intravenous injections of $0.1 \mathrm{cc} / \mathrm{kg}$ Zoletil and $0.1 \mathrm{cc} / \mathrm{kg}$ Rompun. The pig's entire skin was scanned with computed tomography, and the maximum epidermal/ dermal thickness was determined to be $<2 \mathrm{~cm}$ (Fig. 1A). By varying the thickness of the lead shielding (1, 2 and 3 $\mathrm{mm})$, film dosimetry determined the appropriate shielding thickness (3 mm) (Fig. 1B). The pig's dorsal skin was divided into four sections. A lead shield containing 11 cut-out squares, $2 \mathrm{~cm} \times 2 \mathrm{~cm}$ in size and at least $2.5 \mathrm{~cm}$ from each other, was placed over one section of the dorsal skin. A single fraction of $15,30,50$ or 75 Gy with $6-\mathrm{MeV}$ electrons was delivered to each section of the dorsal skin using a linear accelerator (Elekta, Stockholm, Sweden), ensuring that $>90 \%$ of the prescribed dose would be limited to a maximum depth of $2 \mathrm{~cm}$ (Fig. 1C-D).

\section{Skin toxicity assessment}

The pig was housed and observed for 12 weeks to allow acute and late effects of radiation to develop. The four squares located in the center of the dorsal skin, each of which was irradiated with a different dose, were left for weekly observation for gross skin changes and spectrophotometric analysis until the 12th week. Radiation Therapy Oncology Group (RTOG) toxicity grading [6] was used for the assessment of the gross skin reaction after irradiation (Table 1). Changes in the skin color were assessed weekly using a portable spectrophotometer (CM-700d, Konica Minolta Sensing Inc., Osaka, Japan), and melanin and erythema indices were recorded. The melanin index $(\mathrm{M})$ and erythema index (E) are defined as:

$\mathrm{M}=100 \times \log$ (1/intensity of reflected red light)

$\mathrm{E}=100 \times \log$ (intensity of reflected red light/intensity of reflected green light) [7]

\section{Immunohistochemical analysis for skin microvasculature}

Two of the 44 fields were biopsied in the 1st, 2nd, 4th, 6th and 9th weeks for each radiation dose. The four fields that were left for observation were biopsied in the 12th week. Each biopsied specimen consisted of a full-thickness block $(1.5 \mathrm{~cm} \times 0.5 \mathrm{~cm})$ within the irradiated field. Half of the block was fixed in $10 \%$ neutral buffered formalin, and the other half was frozen in liquid nitrogen for Western blot analysis. Formalin-fixed biopsy samples were embedded in paraffin, sectioned, and then examined histologically. The $5-\mu \mathrm{m}$ sections from each biopsy specimen were stained with anti-CD31 monoclonal antibody for pig (Abcam, Cambridge, UK) to determine microvessel density according to a standard three-step immunohistochemical procedure [8]. Each section was examined microscopically and the cross-sectional area of the positively stained vessel lumens were measured in five high-power fields (HPF) per slide $(\times 200)$, and average values were obtained. Within-group and between-group means were compared, and significance was detected with a two-tailed $t$ test.

\section{Western blotting for IL-6 and TGF- $\beta 1$}

The frozen tissue samples were homogenized in RIPA lysis buffer (150 mM sodium chloride, $0.5 \%$ Triton X-100, 0.5\% sodium deoxycholate, $0.1 \%$ sodium dodecyl sulfate (SDS), $50 \mathrm{mM}$ Tris, $\mathrm{pH} 8.0$ ) that contained protease inhibitors. The lysates were then centrifuged at $13000 \mathrm{rpm}$ at $4^{\circ} \mathrm{C}$ for $5 \mathrm{~min}$ and separated on SDS-PAGE. The proteins were transferred to a polyvinylidene fluoride (PVDF) membrane (Millipore, MA, USA) and then incubated overnight with antibodies and detected with ECL (ECL Western Blotting Substrate, Pierce, USA) following treatment with 5\% milk powder in Tris buffered saline (TBS) to prevent nonspecific reactions. The specific antibodies used for this experiment were rabbit anti-IL-6 (Abcam, Cambridge, UK), rabbit anti-TGF- $\beta 1$ (Abcam, Cambridge, UK) and mouse anti- $\alpha$-tubulin (Abcam, Cambridge, UK). Each dried blot 

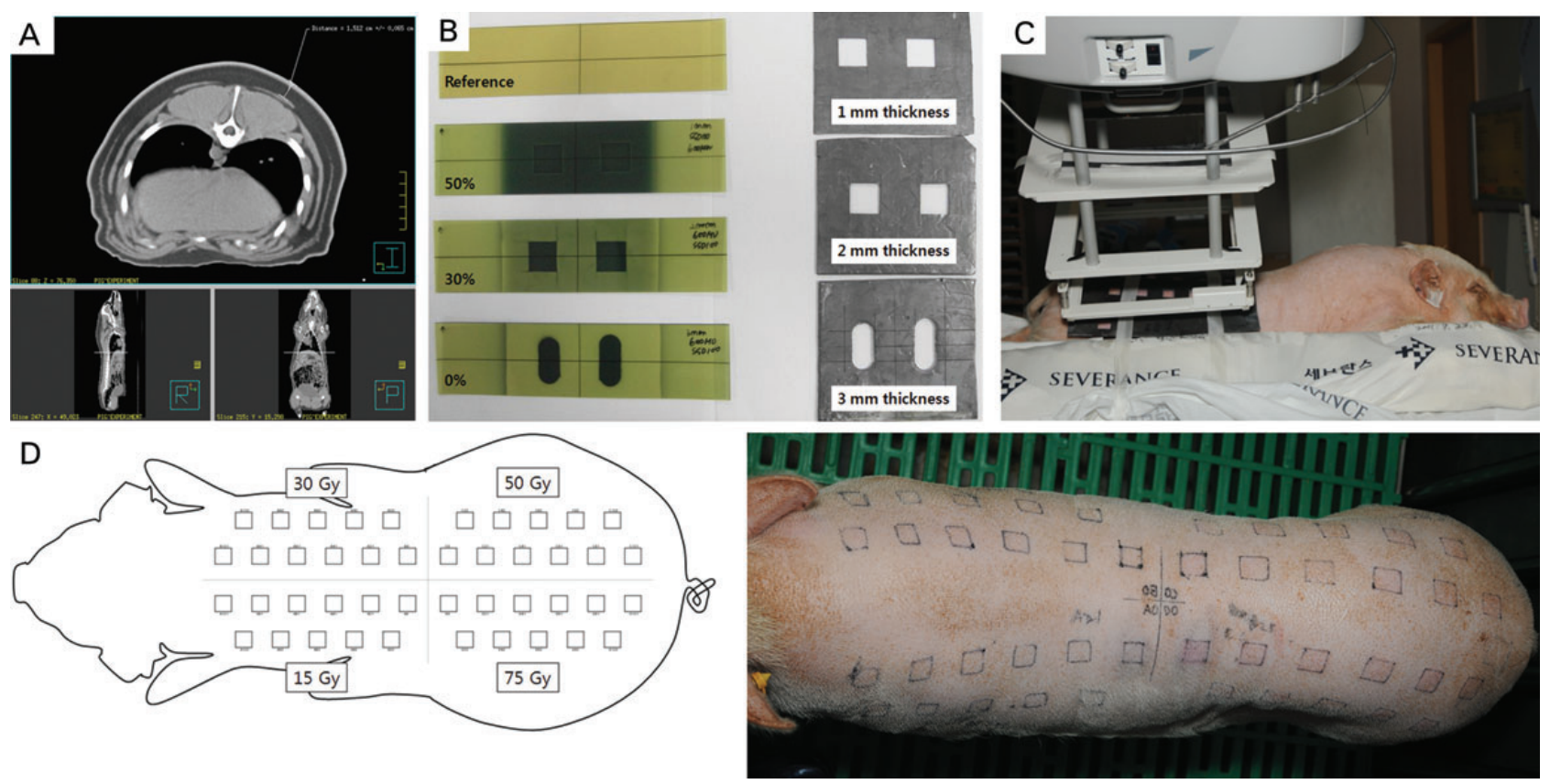

Fig. 1. Experimental design. (A-B) The thickness of the pig's skin was measured using computed tomography, and appropriate thickness $(3 \mathrm{~mm})$ of the lead cutout was determined using film dosimetry. (C-D) The pig's dorsal skin was divided into 4 sections. A lead shield containing 11 cut-out squares, $2 \mathrm{~cm} \times 2 \mathrm{~cm}$ in size and at least $2.5 \mathrm{~cm}$ from each other, was placed over one section of the dorsal skin. A single fraction of $15,30,50$ or $75 \mathrm{~Gy}$ with $6-\mathrm{MeV}$ electrons was delivered to each section of the dorsal skin using a linear accelerator.

Table 1. Radiation Therapy Oncology Group toxicity grading

\begin{tabular}{|c|c|c|c|c|c|}
\hline & Grade 0 & Grade 1 & Grade 2 & Grade 3 & Grade 4 \\
\hline Acute & None & $\begin{array}{l}\text { Follicular, faint or dull } \\
\text { erythema/epilation/dry } \\
\text { desquamation/decreased } \\
\text { sweating }\end{array}$ & $\begin{array}{l}\text { Tender or bright } \\
\text { erythema, patchy moist } \\
\text { desquamation/moderate } \\
\text { edema }\end{array}$ & $\begin{array}{l}\text { Confluent, moist } \\
\text { desquamation other } \\
\text { than skin folds, pitting } \\
\text { edema }\end{array}$ & $\begin{array}{l}\text { Ulceration, } \\
\text { hemorrhage, } \\
\text { necrosis }\end{array}$ \\
\hline Chronic & None & $\begin{array}{l}\text { Slight atrophy; } \\
\text { pigmentation change; } \\
\text { some hair loss }\end{array}$ & $\begin{array}{l}\text { Patch atrophy; moderate } \\
\text { telangiectasia; total hair } \\
\text { loss }\end{array}$ & $\begin{array}{l}\text { Marked atrophy; gross } \\
\text { telangiectasia }\end{array}$ & Ulceration \\
\hline
\end{tabular}

was scanned and saved as a TIFF file, and the density of the corresponding band was quantified using Image $\mathrm{J}$, a Java-based image processing software (US National Institutes of Health, Bethesda, Maryland; downloaded from http://imagejnihgov/ij/) [9]. Values are expressed as the relative intensity to $\alpha$-tubulin.

\section{RESULTS}

\section{Gross and histologic changes of the skin}

Figure 2 summarizes the gross and histologic changes of the skin after a single fraction irradiation of $15 \mathrm{~Gy}, 30 \mathrm{~Gy}$, $50 \mathrm{~Gy}$ or $75 \mathrm{~Gy}$, observed at 0-12 weeks post-irradiation. Transient erythema occurred within 1 week in the fields irradiated with 50 and $75 \mathrm{~Gy}$, and it was followed by a 4-week period of latency during which no definite skin reaction was observed. Erythema and dry desquamation (RTOG Grade 1), moist desquamation (Grade 3), and ulceration (Grade 4) appeared 4, 6 and 9 weeks after irradiation, respectively. Fields receiving $15 \mathrm{~Gy}$ and $30 \mathrm{~Gy}$ healed without ulceration, while irradiation $\geq 50 \mathrm{~Gy}$ resulted in ulceration followed by necrosis (Fig. 2A). A plot of gross skin changes according to the RTOG grading of acute skin toxicity vs time after irradiation showed skin changes were more pronounced and occurred early with increasing radiation dose (Fig. 2D). Figure 2 B-C shows histologic evaluation of full-thickness biopsy specimens after $30 \mathrm{~Gy}$ (H\&E, magnification $\times 100$ and $\times 400$, respectively). The changes in the epidermal layer corresponded to those of the gross skin. Interestingly, the average number of eosinophils per HPF rose sharply after 4 weeks, peaked at 7-8 weeks and disappeared at 12 weeks post-irradiation. Degranulation 


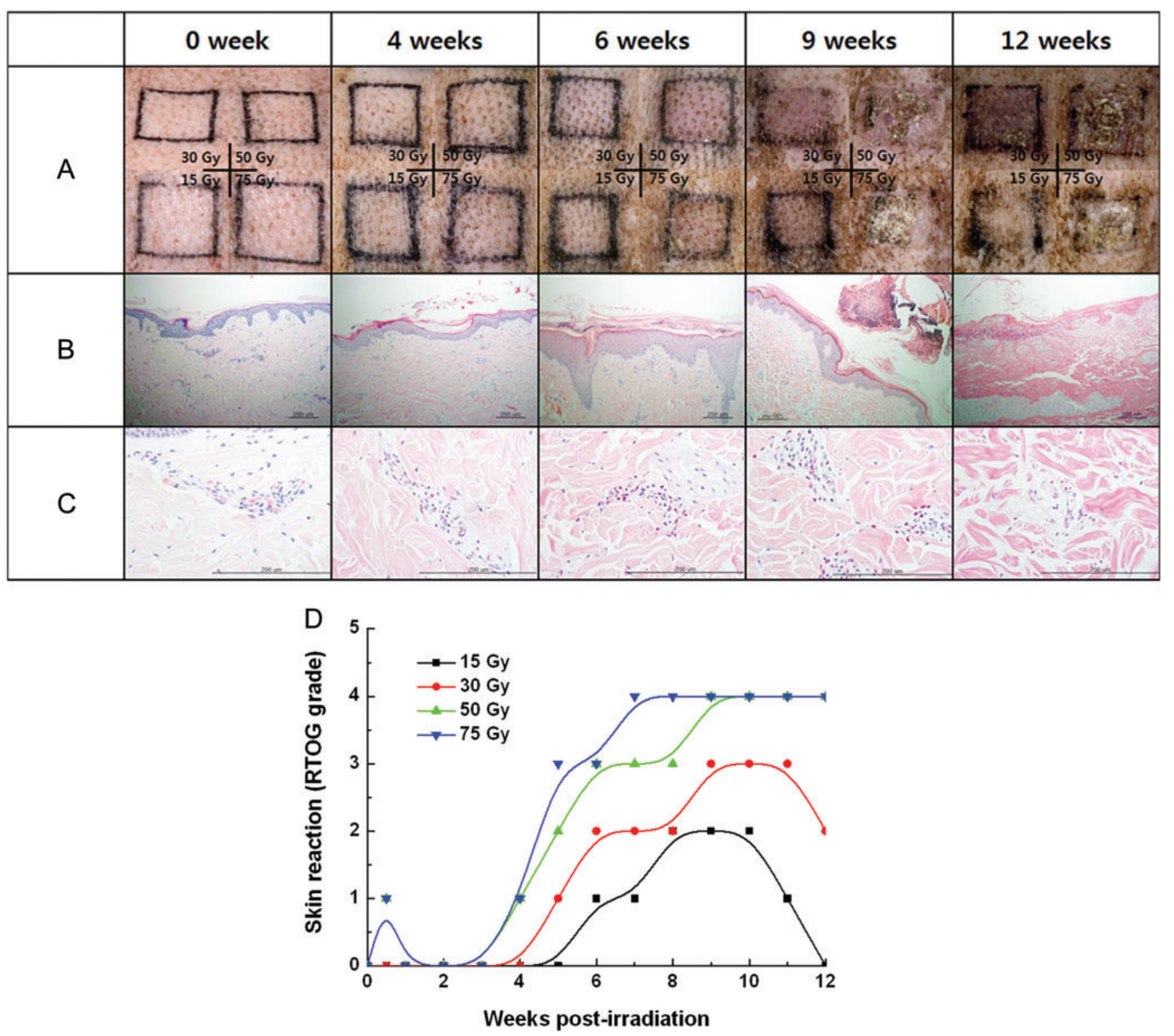

Fig. 2. (A) Gross skin changes in 15, 30,50 and 75 Gy fields. Erythematous changes, wet desquamation and ulceration began to appear 4, 6 and 9 weeks after irradiation, respectively. (B-C) Histologic evaluation of full-thickness biopsy specimens after $30 \mathrm{~Gy}$ (H\&E, magnification $\times 100$ and $\times 400$ ). (D) Gross skin changes according to RTOG grading of acute skin toxicity are plotted against time after irradiation.

from eosinophils was apparent 9 weeks after irradiation. Figure 3 shows patterns of eosinophil infiltration in the intra- and perivascular spaces of the dermis biopsied 4 weeks (A) and 9 weeks (B) after 30-Gy irradiation, as well as the average number of eosinophils in five HPF (magnification, $\times 400$ ) from tissue sections irradiated with 15-75 Gy (C). The rate of increase and the peak in the eosinophil count was markedly pronounced after $75 \mathrm{~Gy}$. Induction of eosinophilia seemed to coincide with the acute and intermediate skin response to radiation.

\section{Spectrophotometry}

Figure 4 shows changes in the mean values of melanin and erythema indices in the porcine skin that received 15 and $30 \mathrm{~Gy}$, from the time of irradiation to 12 weeks post- irradiation. The melanin indices for $15 \mathrm{~Gy}$ began increasing sharply from 0.35 at 6 weeks to a peak value of 0.95 at 10 weeks, and decreased to a baseline level of 0.43 at 12 weeks post-irradiation. The melanin indices for $30 \mathrm{~Gy}$ were $0.29,0.80$ and 1.04 at 6,10 and 12 weeks, respectively. The pattern of changes in melanin indices closely resembled progression of gross changes in the skin after high-dose ( $\geq 50 \mathrm{~Gy}$ ) irradiation. The erythema indices for the $15 \mathrm{~Gy}$ showed early and continuously increased from 0.8 at 1 week to a peak of 2.1 at 9 weeks, then decreased to 1.8 at 12 weeks post-irradiation. Changes in the erythema indices for 30 Gy showed a close resemblance to those of $15 \mathrm{~Gy}$. This result was in sharp contrast to that of the gross skin reactions, which did not show erythematous changes until 4 weeks after irradiation. The area of skin irradiated with 50 and 75 Gy showed ulceration and necrosis 6 weeks after 

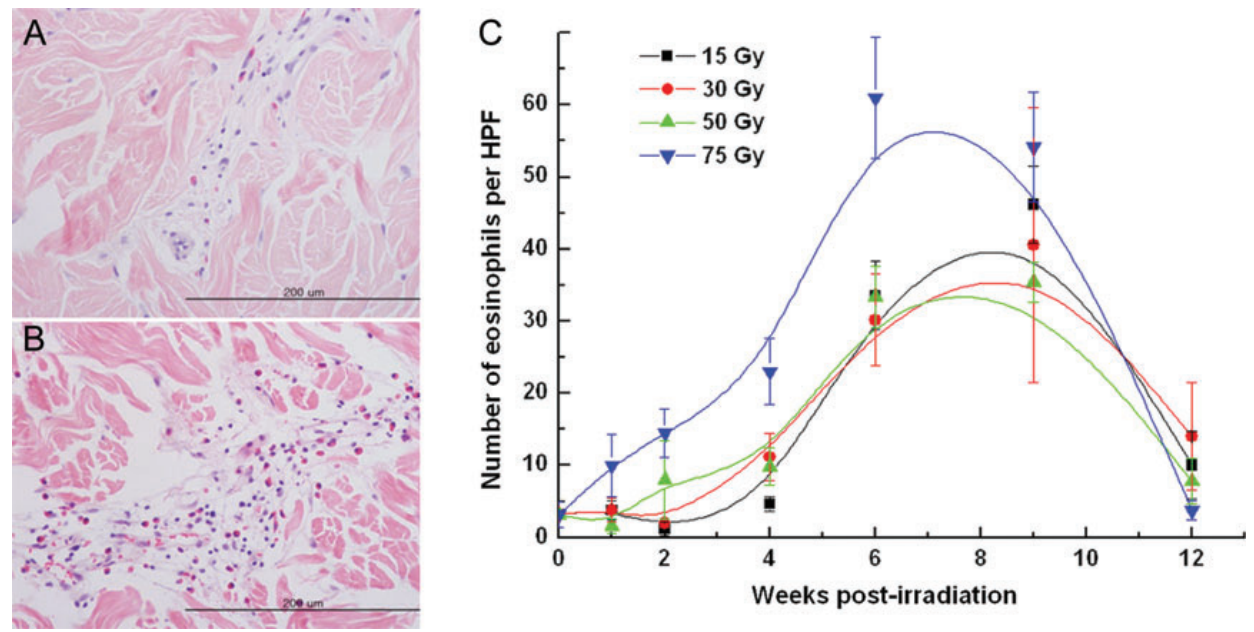

Fig. 3. Correlation between radiation dose and number of eosinophils in the dermis. Representative sections from the skin irradiated with $30 \mathrm{~Gy}$, biopsied (A) 4 weeks after irradiation, and (B) 9 weeks after irradiation (magnification, $\times 400$ ). (C) The mean numbers of eosinophils in 5 high-powered fields (magnification, $\times 400$ ) from a tissue section irradiated with 15-75 Gy are plotted against time.
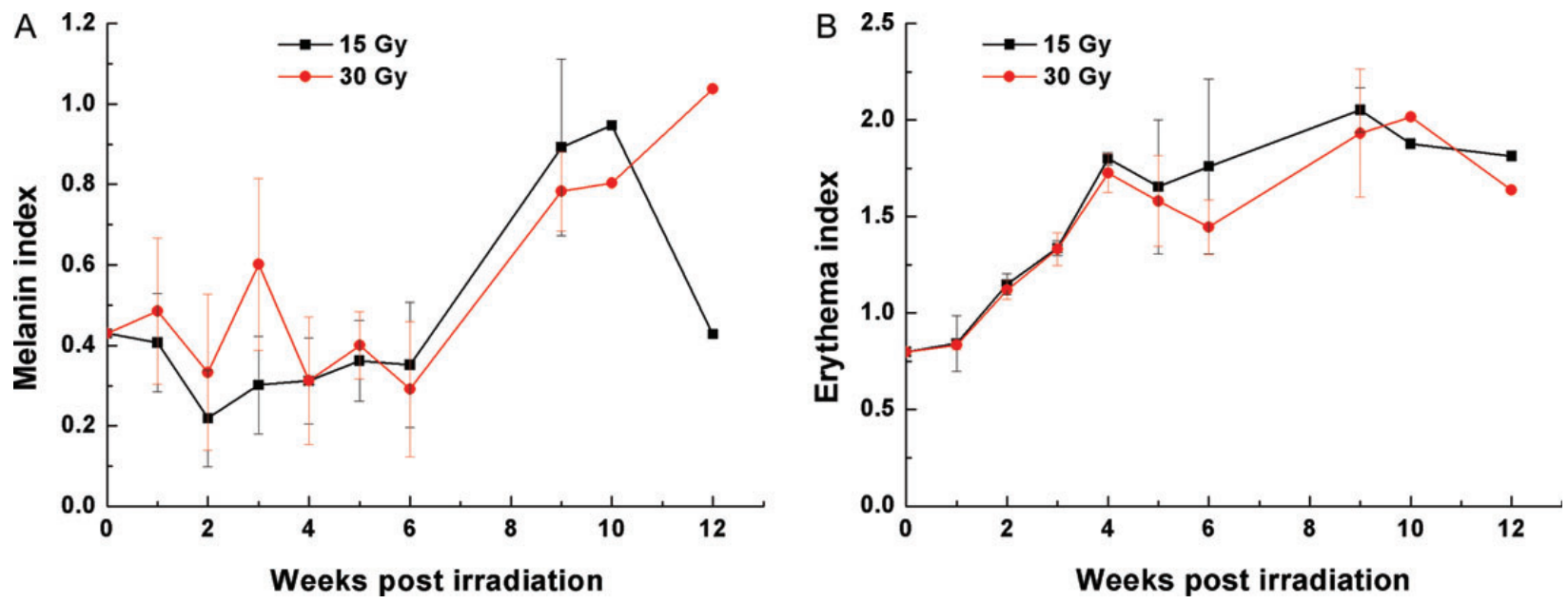

Fig. 4. Spectrophotometry data. (A) The mean value of melanin indices is plotted against time post-irradiation. Melanin indices began rising 6 weeks after irradiation. (B) The mean value of erythema indices is plotted against time post-irradiation. Erythema indices began rising soon after irradiation and continued to increase.

irradiation and was not available for spectrophotometric analysis.

\section{Microvessel density - CD31}

Figure 5 shows changes in the density of dermal microvessels after irradiation with 15-75 Gy. Microvessel density showed a biphasic pattern with a transient peak at 1 week, a nadir at 4-6 weeks, and maximum recovery at 9 weeks. The microvessel density in the 15-Gy field showed full recovery 12 weeks after irradiation. On the other hand, microvessel density in the 30-75 Gy fields recovered to maximum values at 9 weeks post-irradiation and decreased towards the end of observation. Microvessel density from 50- and 75-Gy fields showed lower initial transient peaks and lower nadirs at earlier time points compared with the values from 15- and 30-Gy fields. The initial peaks (at 1 week post-irradiation) for microvessel density showed significant differences $(P<0.0001)$ between each experimental group irradiated with different doses, while the differences in the nadir (at 4 weeks post-irradiation) for different dose levels were not significant $(P=0.660)$. During the latency period of 0-4 weeks, the changes in the microvessel density were substantial.

\section{Western blot assay}

Figure 6 shows sequential expression of IL-6 (A-B) and TGF- $\beta 1$ (C-D) after irradiation of 15-30 Gy. Expression of IL-6 and TGF- $\beta 1$ increased after $30-G y$ irradiation as 
A

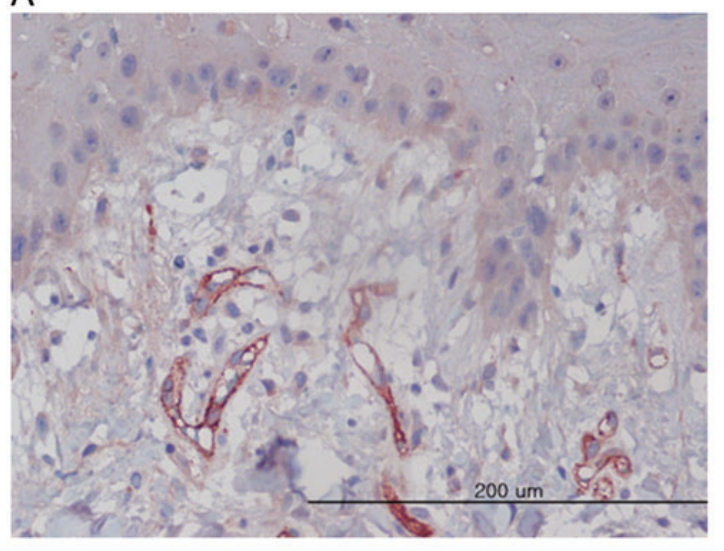

B

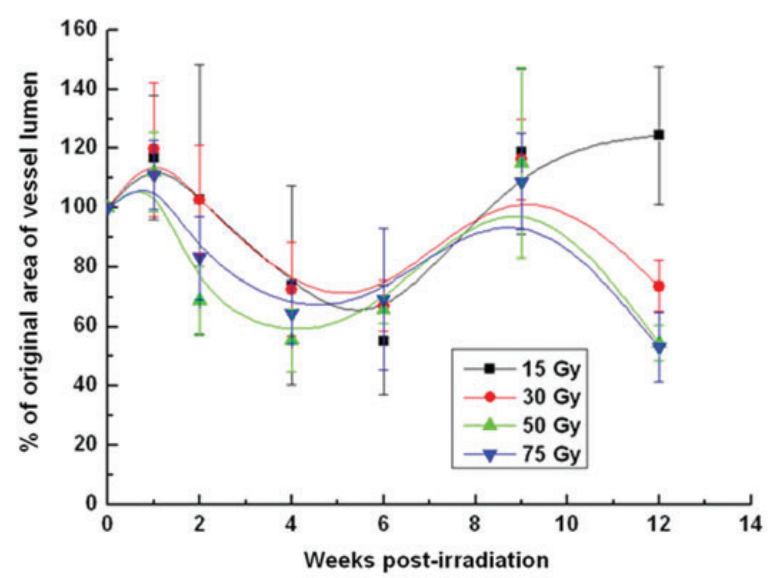

Fig. 5. Changes in $\%$ of original area of microvessel lumen per high-power field after 15-75 Gy irradiation. (A) A representative section from the skin irradiated with $30 \mathrm{~Gy}$ and biopsied 6 weeks after irradiation was stained with antibody to CD31 (magnification, $\times 400$ ). (B) The density of CD31-stained small arterioles was determined by summing the luminal areas of microvessels in each high-powered field (magnification, $\times 200$ ). The mean density of 5 HPFs for each section was compared with the baseline value obtained on Day 0 and is expressed as a percentage.

compared with 15-Gy irradiation. Increase in the level of IL-6 for higher dose (30 Gy) irradiation was detected soon after irradiation and had an earlier peak compared with the values for lower dose (15 Gy) irradiation. Levels of TGF- $\beta 1$ began increasing soon after irradiation and persisted throughout the observation period.

\section{DISCUSSION}

Radiation-induced skin changes typically follow welldefined stages of progression and have been subdivided according to various classifications systems [10, 11]. Early radiation effects typically result from loss of the rapidly proliferating basal epithelial stem cells [12]. Within the first week of radiation, some patients may develop a transient erythema, caused by an inflammatory response leading to capillary vasodilatation, increased vascular permeability, and edema [12, 13]. Erythema demarcating the radiation field begins in the second or third week of fractionated radiation (10-20 Gy) and becomes progressively more evident by the third and fourth weeks of treatment [10]. If the total dose to the skin does not exceed $30 \mathrm{~Gy}$, the dry desquamation phase will typically occur in the fourth or fifth week of radiation [14]. If the total delivered dose is $>40 \mathrm{~Gy}$, the erythema phase may be followed by the moist desquamation phase, which has similar histologic features to a second-degree burn [10]. RTOG toxicity criteria categorize gross skin changes rather subjectively, according to which, follicular, faint or dull erythema is defined as the first sign of skin toxicity. However, RTOG toxicity grading is based on qualitative observation of gross changes and may not reflect early changes in the irradiated skin at the molecular level. In our porcine skin model, erythema developed
4 weeks after irradiation, indicating the latency period was longer than in human skin, albeit the sequence of development of radiation-induced changes in pigskin closely resembled that of human skin. To date, no method has been developed to detect and quantify the radiation-induced skin reaction during the early latent period.

A portable narrowband spectrophotometer is a useful tool to quantitatively assess the skin color, and it has been shown that melanin and erythema indices are useful parameters reflecting the differences in skin color [15]. In the current study, erythema indices began to rise from the first week of irradiation and continued to increase until 9 weeks after irradiation. Detection of early changes with a spectrophotometer coincided with early skin response at the histologic and molecular levels, including microvessel density, eosinophilia, and level of IL-6 and TGF- $\beta$ in the irradiated tissue. Further investigation correlating spectrophotometric indices with levels of molecular changes may allow skin spectrophotometry to be used as a non-invasive indicator for early skin reactions to radiation.

Vascular endothelial cells are the primary site of late radiation-induced skin injuries, leading to dermal fibrosis, although endothelial cell damage begins as early as the acute phase skin reaction [12]. We showed that skin damage, indicated by decreased microvessel density, began soon after a transient rise at 1 week, and the damage was most severe at 4-6 weeks post-irradiation, after which the recovery process began. Skin irradiated with 15-30 Gy continued to recover from radiation-induced injury, while skin irradiated with 50-75 Gy was unable to recover fully because the initial damage was too severe. This resembles a consequential late complication in fractionated radiotherapy, which is a late effect consequent to a persistent severe early 
A
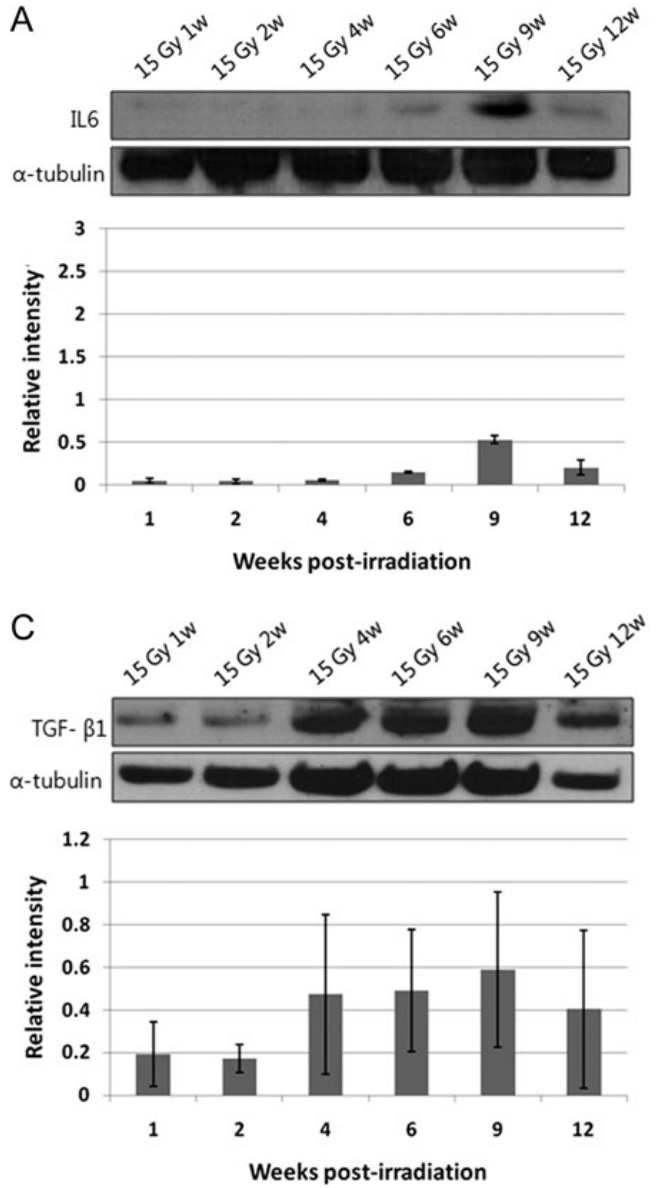

B
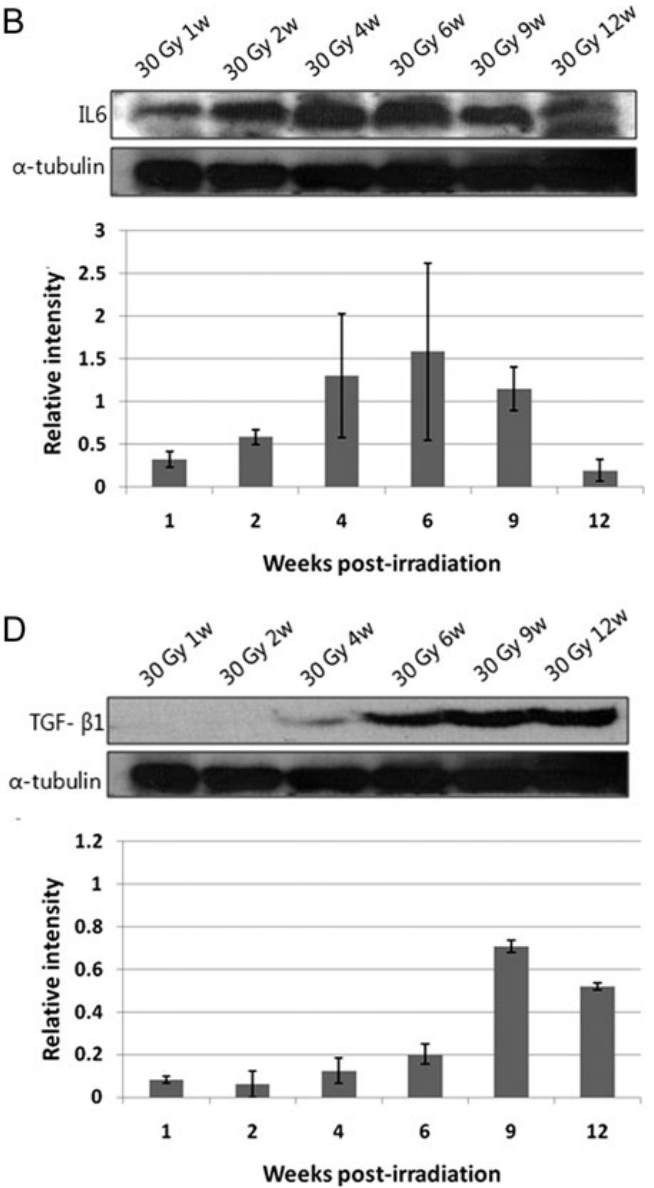

Fig. 6. Protein expression of IL-6 (A-B) and TGF- $\beta 1$ (C-D) after irradiation of 15-30 Gy. Western blot analyses were performed, and density of the corresponding bands was quantified using Image $\mathrm{J} 1.37 \mathrm{v}$ analysis software. Values are expressed as the relative intensity compared with $\alpha$-tubulin.

effect [12]. In a previous investigation, pig dorsal skin irradiated with single fractional electron beams of $16 \mathrm{~Gy}, 18$ Gy and 20 Gy showed a similar pattern of changes in microvessel density [2]. In this experiment, the nadir was reached at 7 weeks post-irradiation, with a higher radiation dose resulting in a lower nadir. In our study, the density curves of high-dose radiation ( $50 \mathrm{~Gy}$ and $75 \mathrm{~Gy}$ ) showed lower nadirs appearing earlier than those of low-dose radiation (15 and $30 \mathrm{~Gy}$ ). We used significantly higher radiation doses compared with the doses used in previous works, and it appears that the higher dose caused earlier skin damage that took longer to heal.

The size of radiation fields used in the current study $(2 \mathrm{~cm} \times 2 \mathrm{~cm})$ is smaller than those from previous studies $[2,16]$. The nadir in microvessel density was reached at 6 weeks after irradiation of $15 \mathrm{~Gy}$. Hadad et al. irradiated pig's skin $(18 \times 40 \mathrm{~cm})$ with a single fractional dose of 16 20 Gy using a $6 \mathrm{MeV}$ electron beam, and the nadir in microvessel density appeared between 7 and 10 weeks. Current practice of radiotherapy accepts the notion that normal skin tolerance is field-size dependent. The relatively similar rates of skin damage, despite the large difference in field sizes between the two experiments, may be explained by the observation made by Hopewell et al., where irradiation of skin fiddles of $16 \mathrm{~cm}^{2}(4 \mathrm{~cm} \times 4 \mathrm{~cm})$ and $64 \mathrm{~cm}^{2}$ $(16 \mathrm{~cm} \times 4 \mathrm{~cm})$ showed no significant difference between the two field sizes [16]. It may be possible that the rate of skin damage is not so field-size dependent as the rate of healing is, and further studies are required to determine the correlation between the healing rate and the size of the radiation field.

Gross skin changes according to RTOG criteria in Fig. 2A showed clear dose-dependence of radiation-induced skin damage. However, the dose-dependent response was not as clear at the histologic and molecular levels. Although significant changes in the gross skin were not detected during the first 4 weeks after irradiation, eosinophil counts began rising as early as 1 week after irradiation. Changes in the eosinophil counts showed dose-dependent relationships during the first 4 weeks: eosinophil counts began rising soon after irradiation of 75 Gy while 15 Gy-irradiation did not cause an increase in eosinophil counts until the 4th 
week. For the changes in microvessel density, the nadirs for 50 Gy and 75 Gy appeared earlier than those for 15 Gy and $30 \mathrm{~Gy}$, and the level of microvessel density indicated recovery from radiation damage at 12 weeks after $15 \mathrm{~Gy}$ and 30 Gy, but not after 50 Gy and 75 Gy. There is a clear difference in gross skin reactions as well as changes at histologic and microscopic levels between irradiation of 15-30 Gy and irradiation of 50-75 Gy. A single fraction of $50 \mathrm{~Gy}$ can be considered a threshold dose for our porcine skin model, and further studies are required to determine clearer dosedependence for doses lower than $50 \mathrm{~Gy}$.

In the early stages of endothelial cell damage, leukocytes infiltrate into the irradiated tissue and induce inflammatory responses and early fibrotic changes through secretion of various cytokines including TGF- $\beta$ and IL-6 [4]. The process of late fibrosis mediated by endothelial cell damage is not clearly understood. Eosinophil granules contain fibrogenic molecules, including TGF- $\beta$, IL-4, IL-6, TNF- $\alpha$, eosinophil cationic protein, and major basic protein. It had been demonstrated that eosinophils act as direct modulatory cells in fibroblast proliferation, collagen synthesis, and lattice contraction, in part through TGF- $\beta$ [17]. In the current study, eosinophils began to appear at 2 weeks, increased rapidly in number at 4 weeks, peaked at 6-9 weeks, and disappeared 12 weeks after irradiation. This pattern is closely followed by the level of TGF- $\beta 1$ expression in the Western-blot analysis, which was detected 1 week after irradiation and continued to increase afterward. Unlike normal wound healing where feedback mechanisms tightly regulate activation of fibroblasts into myofibroblasts and in turn proliferation and deposition of the collagen matrix, fibrosis is characterized by sustained activation of myofibroblasts through abnormal production of stimulating factors, particularly of TGF- $\beta 1$. TGF- $\beta 1$ is considered a master switch for the fibrotic changes of the skin after exposure to radiation [18]. Martin et al. [19] found that the TGF- $\beta 1$ mRNA level was increased in the irradiated skin during the early erythematous phase, which started 3 weeks after irradiation, as well as during the later phases of fibrosis, from 6-12 months after irradiation. They suggested that TGF- $\beta 1$ is one of the key cytokines involved in the cascade of events that leads to radiation-induced fibrosis, at both early and late stages. The role of eosinophils and cytokines produced from eosinophil degranulation, including TGF- $\beta 1$, in radiation-induced fibrosis is unclear. Nonetheless, the active transition in the molecular environment of the skin during the latency period, including microvessel density, eosinophil level, and the level of TGF- $\beta 1$ and IL-6, provides grounds for early intervention for the prevention and treatment of radiation-induced skin injury.

It is a common practice to divide radiation-induced normal tissue injury into acute and late phases. However, it is clearly evident that response to radiation is not a combination of discrete and unrelated episodes but rather a continuous course of interrelated events. Our porcine skin model revealed that the early latency period after high-dose irradiation is not a static phase suggested by the lack of gross changes but rather an active period during which many molecular events are in progress. Further studies are planned to determine the effects of early therapeutic intervention on the modulation of acute radiation-induced skin toxicity and development of dermal fibrosis.

In the current study, we designed a large number of treatment fields that are under the influence of identical host factors, thus allowing fast and reproducible results by varying external treatment conditions. We established a time-dose response relationship for the skin exposed to single-fraction high-dose radiation using spectrophotometric analysis, eosinophil counts, and molecular indicators including CD31-stained microvascular density and the level of IL- 6 and TGF- $\beta 1$ in the irradiated tissue.

The current study was designed to determine appropriate biomolecular markers that can be used as indicators of radiation-induced skin damage as well as the maximum dose that the porcine skin can tolerate for further experiments on radiation-induced skin toxicity. Results of the current study indicated that many histologic and molecular events occurred during the early latent period (first 4 weeks after irradiation), and intervention during this period may modulate the course of skin injury after irradiation. We plan to conduct further experiments on radiation-induced skin injuries and mitigating factors using our porcine model.

\section{ACKNOWLEDGEMENTS}

The abstract of the current study was accepted for poster viewing at the 54th Annual Meeting of the American Society for Radiation Oncology (ASTRO), Boston, MA, October 2012.

\section{CONFLICT OF INTEREST}

The authors declare that there are no conflicts of interest.

\section{FUNDING}

This work was supported by a faculty research grant from the Yonsei University College of Medicine for 2010 (6-2010-0152) and by a National Research Foundation of Korea (NRF) grant funded by the Korean government (MEST) (No. 2011-0031695).

\section{REFERENCES}

1. Hoppe BS, Laser B, Kowalski AV et al. Acute skin toxicity following stereotactic body radiation therapy for stage I non-small-cell lung cancer: who's at risk? Int J Radiat Oncol Biol Phys 2008;72:1283-6. 
2. Hadad I, Johnstone BH, Brabham JG et al. Development of a porcine delayed wound-healing model and its use in testing a novel cell-based therapy. Int J Radiat Oncol Biol Phys 2010;78:888-96.

3. Archambeau JO, Pezner R, Wasserman T. Pathophysiology of irradiated skin and breast. Int J Radiat Oncol Biol Phys 1995;31:1171-85.

4. Lawenda BD, Johnstone PA. Skin. In: Shrieve DC, Loeffler JS (eds). Human Radiation Injury. Philadelphia, PA: Lippincott Williams \& Wilkins, 2011, 499-515.

5. Morris GM, Hopewell JW. Cell kinetic changes in the follicular epithelium of pig skin after irradiation with single and fractionated doses of X rays. Brit J Radiol 1989;62:41-7.

6. Cox JD, Stetz J, Pajak TF. Toxicity criteria of the Radiation Therapy Oncology Group (RTOG) and the European Organization for Research and Treatment of Cancer (EORTC). Int J Radiat Oncol Biol Phys 1995;31:1341-6.

7. Draaijers LJ, Tempelman FR, Botman YA et al. Colour evaluation in scars: tristimulus colorimeter, narrow-band simple reflectance meter or subjective evaluation? Burns 2004;30:103-7.

8. Lamme EN, de Vries HJ, van Veen $\mathrm{H}$ et al. Extracellular matrix characterization during healing of full-thickness wounds treated with a collagen/elastin dermal substitute shows improved skin regeneration in pigs. J Histochem Cytochem 1996;44:1311-22.

9. Rasband W. ImageJ. http://imagejnihgov/ij/. (1 January 2012, date last accessed).
10. Fajardo L. Skin Pathology of Radiation Injury. New York: Masson Publishing, 1982.

11. Tessmer C. Radiation Effects in Skin. Baltimore: Williams \& Wilkins, 1971.

12. Hall E. Radiobiology for the Radiobiologist. 7th edn. Philadelphia: Lippincott Williams \& Wilkins, 2012.

13. Jolles B, Harrison RG. Enzymic processes and vascular changes in the skin radiation reaction. Brit J Radiol 1966;39:12-8.

14. Rubin P, Casarett G. Clinical Radiation Pathology. Vol. 2. Philadelphia: W.B Saunders, 1968.

15. Yun IS, Lee WJ, Rah DK et al. Skin color analysis using a spectrophotometer in Asians. Skin Res Technol 2010; 16:311-5.

16. Hopewell JW, Young CM. The effect of field size on the reaction of pig skin to single doses of X rays. Brit $J$ Radiol 1982;55:356-61.

17. Levi Schaffer F, Garbuzenko E, Rubin A et al. Human eosinophils regulate human lung- and skin-derived fibroblast properties in vitro: a role for transforming growth factor beta (TGF-beta). Proc Natl Acad Sci U S A 1999;96:9660-5.

18. Martin M, Lefaix J, Delanian S. TGF-beta1 and radiation fibrosis: a master switch and a specific therapeutic target? Int J Radiat Oncol Biol Phys 2000;47:277-90.

19. Martin M, Lefaix JL, Pinton $P$ et al. Temporal modulation of TGF-beta 1 and beta-actin gene expression in pig skin and muscular fibrosis after ionizing radiation. Radiat Res 1993;134:63-70. 